UCRL-JC-124616

PREPRINT

\title{
Positron Beam Lifetime Spectroscopy at Lawrence Livermore National Laboratory
}

\author{
R. Howell \\ T. Cowan \\ J. Hartley \\ P. Stern
}

This paper was prepared for submittal to the

\section{4th International Conference on the Application of Accelerators in Research and Industry \\ Denton, $T X$ \\ November 6-9, 1996}

October 1996

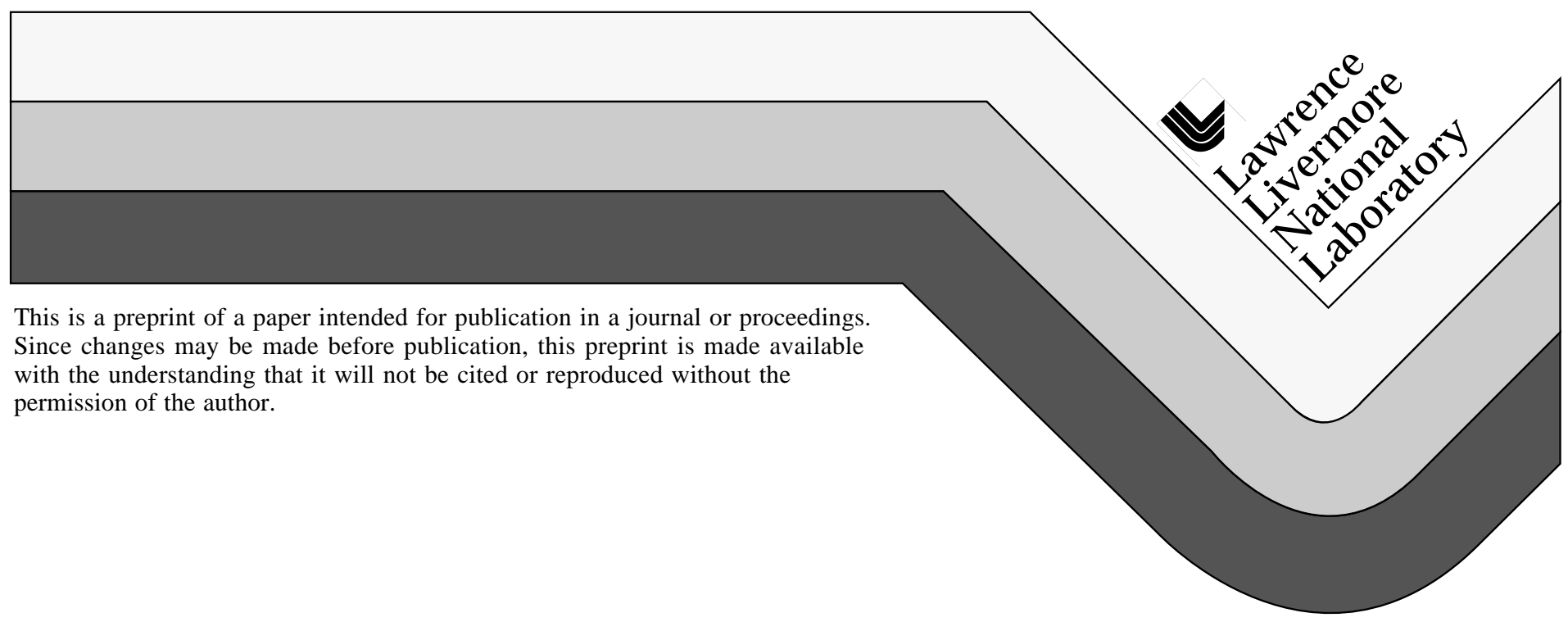




\section{DISCLAIMER}

This document was prepared as an account of work sponsored by an agency of the United States Government. Neither the United States Government nor the University of California nor any of their employees, makes any warranty, express or implied, or assumes any legal liability or responsibility for the accuracy, completeness, or usefulness of any information, apparatus, product, or process

disclosed, or represents that its use would not infringe privately owned rights. Reference herein to any specific commercial product, process, or service by trade name, trademark, manufacturer, or otherwise, does not necessarily constitute or imply its endorsement, recommendation, or favoring by the United States Government or the University of California. The views and opinions of authors expressed herein do not necessarily state or reflect those of the United States Government or the University of California, and shall not be used for advertising or product endorsement purposes. 


\title{
POSITRON BEAM LIFETIME SPECTROSCOPY AT LAWRENCE LIVERMORE NATIONAL LABORATORY
}

\author{
Richard H. Howell ${ }^{a}$, Thomas E. Cowan ${ }^{a}$, Jay H. Hartley ${ }^{a}$ and Philip A. Sterne ${ }^{a, b}$ \\ a Lawrence Livermore National Laboratory, Livermore CA 94550 \\ b Physics Dept., University of California, Davis CA 95616
}

\begin{abstract}
Defect analysis is needed for samples ranging in thickness from thin films to large engineering parts. We are meeting that need with two positron beam lifetime spectrometers: one on a $3 \mathrm{MeV}$ electrostatic accelerator and the second on our high current linac beam. The high energy beam spectrometer performs positron lifetime analysis on thick sample specimens which can be encapsulated for containment or for in situ measurements in controlled environments. At our high current beam we are developing a low energy, microscopically focused, pulsed positron beam to enable positron annihilation lifetime spectroscopy for defect specific, 3-dimensional maps with sub-micron location resolution. The data from these instruments is interpreted with the aid of first principles calculations of defect specific positron lifetimes.
\end{abstract}

\section{INTRODUCTION}

Our understanding of many important mechanical and electrical properties is limited by our knowledge of the microstructure and defects. There are many techniques that can identify the species and size of impurities. There are few however that can detect open volume defects such as atomic vacancies or voids, fig. 1 . All positron annihilation spectrographic methods are particularly sensitive to determining low concentrations of the class of defects including vacancies, voids and negatively charged defects. This sensitivity stems from the attractive interaction between these defects and positrons which often binds the positron to the defect site.
Using positron annihilation lifetime spectroscopy determines the size or charge of defects in metals, semiconductors and molecular or organic compounds.

Positron annihilation lifetime spectroscopy measures the electron density at the annihilation site. Since the electron density is sensitive to the defect volume there is a distinct correlation between the positron lifetime and defect size and condition. In metals and some compounds the correlation between lifetime and defect size can be calculated from first principles with sufficiently high accuracy to differentiate between major defect classes such as vacancies, vacancy clusters and voids. In the best case the positron lifetime is sensitive to the vacancy volume change associated with a dislocation strain field. 

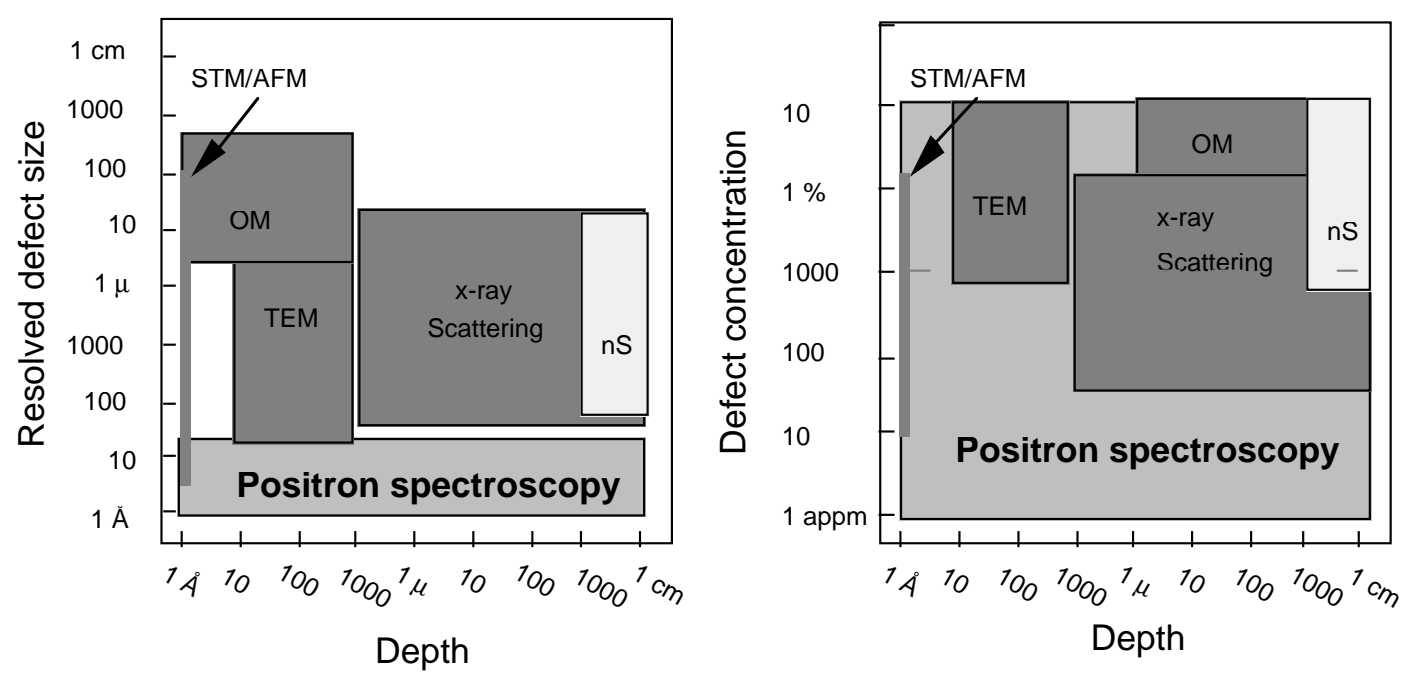

Figure 1. Positron annihilation lifetime analysis fills a special niche for techniques for general vacancy defect analysis, optical microscopy, OM, neutron scattering, nS, transmission electron microscopy, TEM, scanning tunneling microscopy, STM and atomic force microscopy, AFM, and x-ray scattering. Positron techniques are highly sensitive and can resolve the size of atomic vacancies at any depth in a sample.

\section{LABORATORY CAPABILITIES}

Positron annihilation spectroscopies have been traditionally performed by placing the sample in proximity to a radioactive source. This "sourcesample" technique has been used at Livermore to measure electron momenta to determine Fermi surfaces through positron annihilation angular correlation and defect properties by positron annihilation lifetime spectroscopy. Significant advantages can be achieved by performing positron measurements with a monoenergetic beam. There is a relaxation of the sample geometry so that engineering samples can be measured. Better definition of the measurement volume is obtained by controlling the energy and direction of the positrons during implantation in the sample. For high energies the positron beam can even pass through thin windows before implanting into the sample allowing in situ measurements in controlled environments. In positron annihilation lifetime spectroscopy higher data rates are achieved by a positron beam due to the nearly $100 \%$ efficiency in determining the implantation time. Also in a well controlled geometry only annihilation events from the sample will contribute to the data which eliminates systematic contributions from the positron source and simplifies the data analysis.

\section{High Energy Beam}

There are both low and high energy positron beams available at LLNL. Our high energy beam is derived from a $100 \mathrm{mCi}^{22} \mathrm{Na}$ source moderated by a 2 micron thick tungsten single crystal foil positioned in the terminal of a $3 \mathrm{MeV}$ Pelletron electrostatic accelerator, see fig. 2. Both moderated positrons and positrons directly emitted from the source are captured and accelerated. This beam contains a current of $5 \cdot 10^{5} \mathrm{e}^{+} \mathrm{s}^{-1}$ captured directly from the radioactive source at $3 \mathrm{MeV}$. A similar current of moderated positrons can be obtained. To perform positron lifetime spectroscopy the time of implantation is determined for each positron as it passes through a $2 \mathrm{~mm}$ plastic scintillator. Annihilation gammarays of the implanted positrons are detected by a $\mathrm{BaF}_{2}$ detector. The annihilation lifetime is calculated from the time difference in the two detectors with a system resolution of $\sim 250$ ps. Positrons leave the implantation detector with a broad energy distribution, an average energy of 2.6 $\mathrm{MeV}$ and a $1 \mathrm{~cm}$ effective beam diameter at a sample placed $4 \mathrm{~cm}$ down stream. At this energy positrons implant from $\mathrm{mm}$ to $\mathrm{cm}$ into a sample depending on the sample density. Positrons that scatter away from the sample are rejected by an anticoincidence detector. In the pictured close coupled geometry a small, $<1 \%$, contribution from annihilations in the detector system is found in measured spectra and 2000 counts per second can be achieved. The annihilation detector can also be run in coincidence with a second detector to constrain all events to annihilation in the sample. This geometry reduces the counting rates to less than 1000 counts per second This system is modeled on the one used successfully at Stuttgart Germany[1]

We are using positron annihilation lifetime spectroscopy with the high energy beam to determine aging effects in carbon fiber resin 
composites. We are measuring the changes in the hole volume brought on by accelerated aging of the composites at elevated temperature and in hostile atmospheres. Data describing changes in the hole volume are correlated with infrared spectroscopy and mechanical tests to provide a complete description of the changes during aging. We are also determining in radiation damage in metals from induced radiation from high energy accelerator beams and from self irradiation in radioactive species. Interpretation of lifetimes found in the irradiated alloys is aided by first principles calculations of the bulk and defect lifetimes. We are also using the high energy beam to determine changes in polymeric and molecular solids brought on by irradiation or chemical degradation.

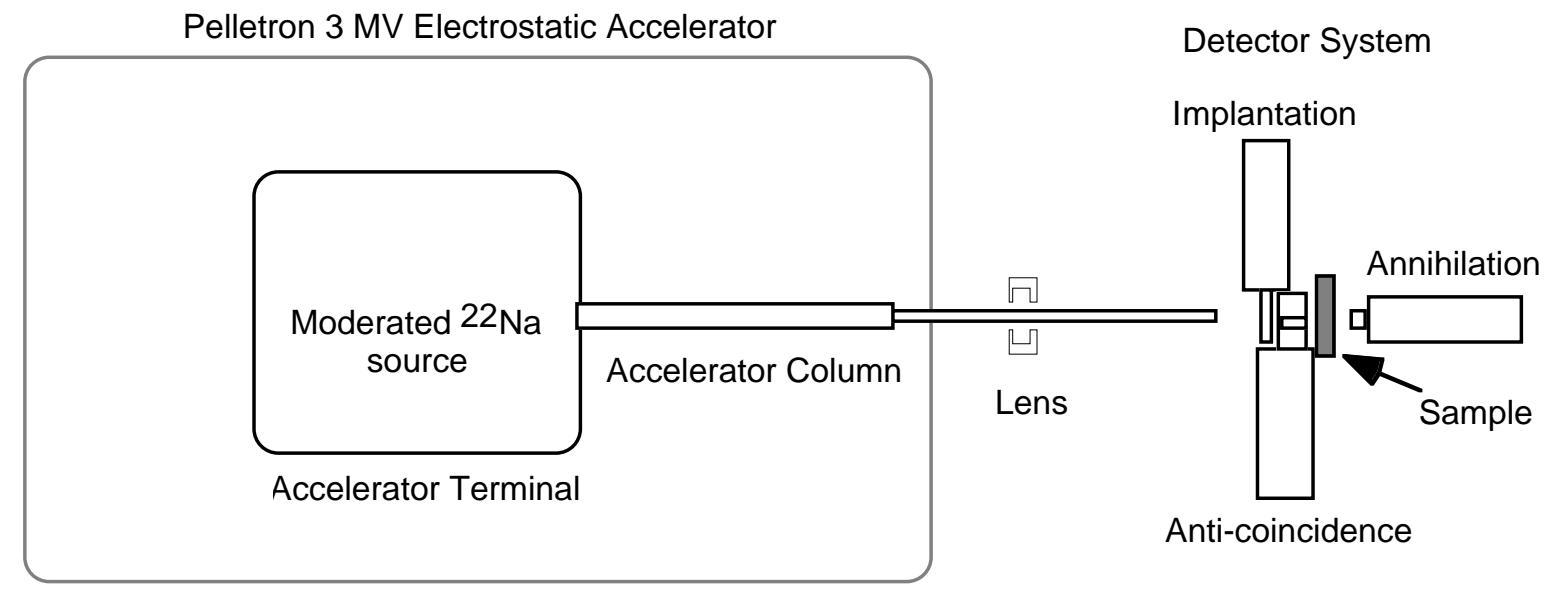

Figure 2. Schematic diagram of the critical components of the high energy beam positron annihilation lifetime system.

\section{3-D Scanning Positron Microprobe}

We are developing a new capability at our high current low energy positron beam located at the LLNL $100 \mathrm{MeV}$ electron linac. By controlling the implantation location and energy of a pulsed, microscopically focused positron beam we will perform positron annihilation lifetime spectroscopy to determine defect concentration and size over spatial volumes of $0.025 \mu \mathrm{m}^{3}$. Our high current positron beam has recently been upgraded to $10^{10} \mathrm{e}^{+} \mathrm{s}^{-1}$. These high initial currents will enable us to perform rapid analysis and 3 dimensional maps of defect characteristics on a practical time scale.

3-Dimensional mapping of defects requires several separate qualities for the positron beam. 3-D spatial sensitivity requires a highly focused, < 1 micron final spot size, and variable energy 1-50 $\mathrm{keV}$ positron energy. Maximum implantation depths for these energies range from 1 to 10 microns depending on sample density. The location of buried features can be located with high precision by sweeping the energy and location of the positron beam in small steps. The size and concentration of the defect species at the end of the positron range are then determined by positron annihilation lifetime spectroscopy measurements correlated to defect size through validated theoretical calculations. To perform positron annihilation lifetime spectroscopy requires pulsing the focused beam with a pulse duration less than 100 ps. To obtain high counting rates our positron pulsing rate will be $15 \mathrm{MHz}$ and multiple detectors will be used for the annihilation gamma rays.

Many of the beam characteristics described above have been obtained in separate systems [2-4] and a design for an efficient, low source strength microprobe has been reported [5]. The LLNL microprobe design integrates new features and those of the published designs to achieve a beam system with improved efficiency and pulse duration. A schematic of the elements of this system is shown in Fig. 3.

In an optical system the initial conditions of the beam entering a pulsing or focusing system limits quality of the system output. In moderated positron systems positrons are expelled into the vacuum by the negative workfunction of a moderator material. Initial conditions are set by the spot size and the distribution of directions and energies of positrons emitted from the material used to thermalize the initial high energy positron source. In focusing systems the overall brightness of the beam limits the final conditions for a lens system. This limitation has been defeated in 
positron optical transport systems by resetting the initial conditions by implanting and thermalizing the positron beam in one or more intermediate steps. At each thermalization step the history of energy and dispersive distributions is lost and new initial conditions are established. This brightness enhancement technique produces highly focused beams with high transmission efficiency. The initial energy spread of the beam is the critical parameter for pulsing systems. The time compression of the pulse is limited only by time of flight spreading from the energy distribution.

The initial linac beam consists of bright pulses of high intensity and short duration. The first step of the 3 dimensional scanning pulsed positron microprobe will be to trade the short time duration for narrow energy. The initial high current linac beam has an energy width of $4 \mathrm{eV}$ in $3 \mu$ s pulses at $300 \mathrm{~Hz} . \quad$ This beam is transported in a magnetic field to a penning trap pulse stretcher which is the first element of the microprobe ( see

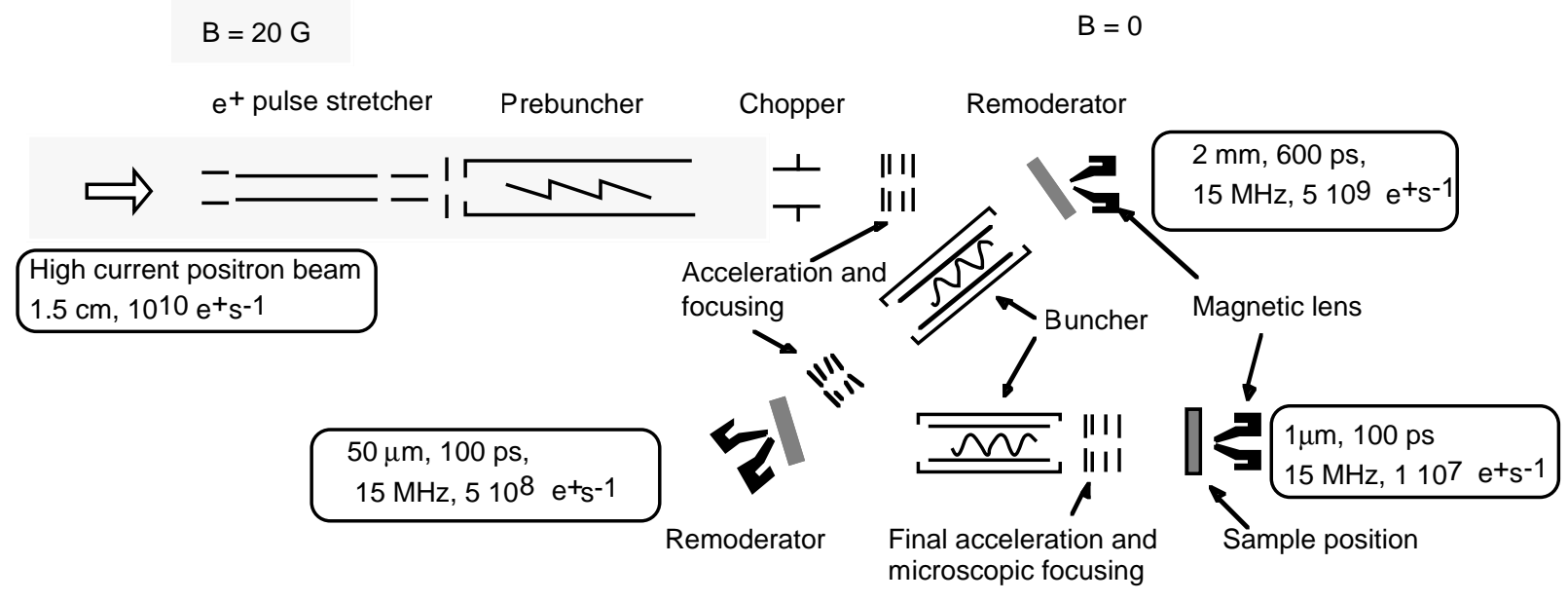

Figure 3 Schematic of the main elements of the 3-D pulsed positron microbeam system. The final beam of more than $10^{7} \mathrm{e}^{+} \mathrm{s}^{-1}$ will be less than 1 micron diameter and 0.5 to $50 \mathrm{keV}$. Beam position will be scanned in the lateral dimensions by magnetic coils and scanned in depth by energy variation to provide a 3-dimensional map of defect concentrations and size.

electrostatic, and single pole magnetic lenses. The beam incident on the first remoderator is expected to be $2 \mathrm{~mm}$ in diameter and have $5 \cdot 10^{9} \mathrm{e}^{+} \mathrm{s}^{-1}$ in $600 \mathrm{ps}$ pulses at $15 \mathrm{MHz}$. The penning trap stretcher, first pulsing section and magnetic filed extraction are

Fig. 3 ). We will trap the positron pulses and slowly raise the trap voltage so that the highest energy positrons will spill over a fixed voltage barrier. The stretcher will convert the $4 \mathrm{eV}, 3 \mu \mathrm{s}$ beam pulse into a $\sim 20 \mathrm{meV}, 300 \mathrm{~ms}$ beam pulse with average energy set by the endcap voltage. This provides a significantly reduced energy distribution for input to the first pulsing electrodes and makes it practical to benefit from an ideal pulsing shape generated by a fast programmable Tektronix AWG2041 waveform generator to obtain high efficiency, sub nanosecond pulses in a single stage. This pulsed beam will then be accelerated to 15 $\mathrm{keV}$ and extracted from the magnetic field of the penning trap. After extraction the beam will be decelerated and focused on the first brightness enhancement moderator by a combination of now fabricated and will be available for test in the near future.

A second stage of focusing and pulsing includes a resonant buncher driven by a Rohde \& Schwartz SMT02 sine wave generator that has been phase and frequency locked to the waveform input of the first pulsing section. This stage completes the pulse compression to $100 \mathrm{ps}$ and focuses the beam on a second remoderator which serves as the input to the microprobe acceleration column. The beam at this point is expected to have 50 micron diameter and $5 \cdot 10^{8} \mathrm{e}^{+} \mathrm{s}^{-1}$. Following the second remoderator is a final stage similar to published designs [6] with variable acceleration and focusing to a one micron beam spot to reach the final beam characteristics of $1 \cdot 10^{7} \mathrm{e}^{+} \mathrm{s}^{-1}, 1-50 \mathrm{keV}, 15 \mathrm{MHz}, 100 \mathrm{ps}$. 
When completed we anticipate using the 3D scanning defect microprobe to study defect distributions in metals and alloys, in inhomogenous systems such as semiconductor devices, and in thin film and composite polymeric materials. With the microprobe we will determine the spatial defect distributions in 3 dimensions near microscopic features such as grain boundaries, cracks, precipitates, and buried interfaces.

\section{ACKNOWLEDGEMENTS}

We wish to thank Drs. W. Triftshäuser, G. Kögel, P. Sperr, and D.T. Suzuki for their valuable conversations on beam pulsing and focusing. This work was performed under the auspices of the US Department of Energy by LLNL under contract No. W-7405-ENG-48.

\section{REFERENCES}

1. W. Bauer, J. Briggmann, H.-D. Carstanjen, S. H. Connell, W. Decker, J. Diehl, K. Maier, J. Major, H. E. Schaefer, A. Seeger, H. Stoll and E. Widmann, Nucl. Inst. and Meth. B50 , 300 (1990)

2. P. Willutzki, J. Störmer, G. Kögel, P.Sperr, D. T. Britton, R. Steindl and W. Triftshäuser, Materials Science Forum 175-178, 237 (1995)

3. R. Suzuki, T. Mikado, M. Chigaki, H. Ohgaki, and T. Yamazaki Applied Surface Science $\underline{85}, 87$ (1995)

4. G.R. Brandes, K. F. Canter, T. N. Horsky and P.H. Lippel and A. P. Mills, Rev. Sci. Instrum. $\underline{59}$, 228 (1988)

5. A. Zecca, R. S. Brusa, M. P. Duarte-Naia, G. PP Karwasz, J. Paridaens, A. Piazza, G. Kögel, P. Sperr, D. T. Britton, K. Uhlmann, P. Willutzki and W. Triftshäuser, Europhys. Lett. 29, 617 (1995)

6. K. Uhlmann, D. T. Britton and G. Kögel, Meas. Sci. Technol. $\underline{6}, 932$ (1995) 



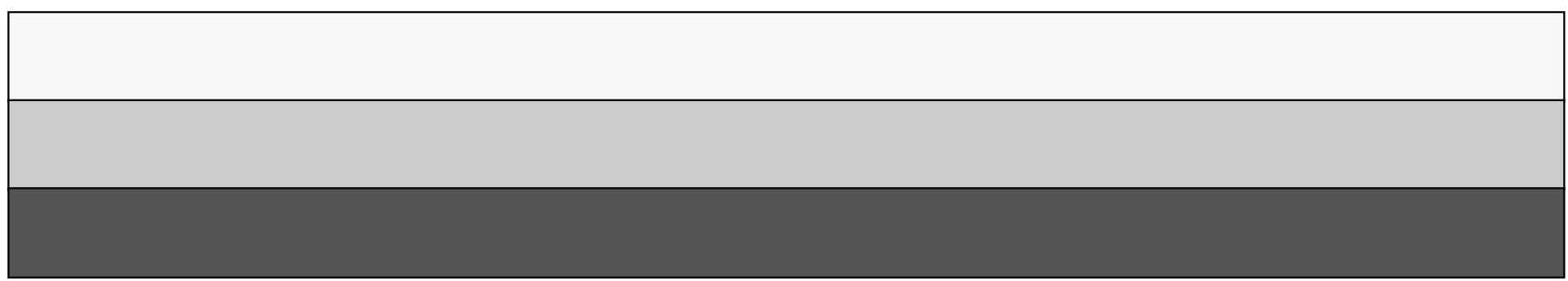

